\title{
Genotype-Informed Versus Empiric Management Of VirEmia (GIVE MOVE): study protocol of an open-label randomised clinical trial in children and adolescents living with HIV in Lesotho and Tanzania
}

Jennifer Anne Brown ${ }^{1,2,3}$, Isaac Ringera ${ }^{4}$, Ezekiel Luoga ${ }^{5}$, Molisana Cheleboi ${ }^{6}$, Namvua Kimera $^{5}$, Josephine Muhairwe ${ }^{4}$, Buntshi Paulin Kayembe ${ }^{7}$, Mosa Molapo Hlasoa ${ }^{7}$, Lorraine Kabundi ${ }^{7}$, Ching Wey David Yav Buoang Mothobi ${ }^{4}$, Lineo Thahane ${ }^{7,8}$, Alain Amstutz ${ }^{1,3,9}$, Nadine Bachmann ${ }^{1,3}$, Getrud Joseph Mollel ${ }^{5}$, Moniek Bresser ${ }^{1,3}$, Tracy Renée Glass ${ }^{1,3}$, Daniel Henry Paris ${ }^{1,3}$, Thomas Klimkait ${ }^{2,3}$, Maja Weisser ${ }^{1,3,5,9}$ and Niklaus Daniel Labhardt ${ }^{1,3,9^{*}}$ (D)

\begin{abstract}
Background: Globally, the majority of people living with HIV have no or only limited access to HIV drug resistance testing to guide the selection of antiretroviral drugs. This is of particular concern for children and adolescents, who experience high rates of treatment failure. The GIVE MOVE trial assesses the clinical impact and cost-effectiveness of routinely providing genotypic resistance testing (GRT) to children and adolescents living with HIV who have an unsuppressed viral load (VL) while taking antiretroviral therapy (ART).

Methods: GIVE MOVE is an open-label randomised clinical trial enrolling children and adolescents ( $\geq 6$ months to $<19$ years) living with HIV with a VL $\geq 400$ copies $/ \mathrm{mL}(\mathrm{c} / \mathrm{mL}$ ) while taking first-line ART. Recruitment takes place at sites in Lesotho and Tanzania. Participants are randomised in a 1:1 allocation to a control arm receiving the standard of care (3 sessions of enhanced adherence counselling, a follow-up VL test, continuation of the same regimen upon viral resuppression or empiric selection of a new regimen upon sustained elevated viremia) and an intervention arm (GRT to inform onward treatment). The composite primary endpoint is the occurrence of any one or more of the following events during the 36 weeks of follow-up period: i) death due to any cause; ii) HIV- or ARTrelated hospital admission of $\geq 24 \mathrm{~h}$ duration; iii) new clinical World Health Organisation stage 4 event (excluding (Continued on next page)
\end{abstract}

\footnotetext{
* Correspondence: n.labhardt@swisstph.ch

'Swiss Tropical and Public Health Institute, Basel, Switzerland

${ }^{3}$ University of Basel, Basel, Switzerland

Full list of author information is available at the end of the article
} licence and your intended use is not permitted by statutory regulation or exceeds the permitted use, you will need to obtain permission directly from the copyright holder. To view a copy of this licence, visit http://creativecommons.org/licenses/by/4.0/. The Creative Commons Public Domain Dedication waiver (http://creativecommons.org/publicdomain/zero/1.0/) applies to the data made available in this article, unless otherwise stated in a credit line to the data. 


\begin{abstract}
(Continued from previous page)
lymph node tuberculosis, stunting, oral or genital herpes simplex infection and oesophageal candidiasis); and iv) no documented $\mathrm{VL}<50 \mathrm{c} / \mathrm{mL}$ at 36 weeks follow-up. Secondary and exploratory endpoints assess additional healthrelated outcomes, and a nested study will assess the cost-effectiveness of the intervention. Enrolment of a total of 276 participants is planned, with an interim analysis scheduled after the first 138 participants have completed follow-up.

Discussion: This randomised clinical trial will assess if the availability of resistance testing improves clinical outcomes in children and adolescents with elevated viremia while taking ART.

Trial registration: This trial is registered with ClinicalTrials.gov (NCT04233242; registered 18.01.2020). More information: www.givemove.org.

Keywords: HIV, Genotypic resistance testing, Drug resistance, Randomised clinical trial, Antiretroviral therapy, Treatment failure, Children, Adolescents, Sub-Saharan Africa
\end{abstract}

\section{Background}

Almost three million children and adolescents worldwide are living with HIV [1]. Every day, almost 1000 children and adolescents are newly infected and over 300 die from HIV/AIDS-related causes [1]. Eastern and Southern Africa are particularly affected, accounting for $65 \%$ of the epidemic in children and adolescents [1]. While substantial progress has been made towards providing antiretroviral therapy (ART) to all people living with HIV, which can suppress viral replication and prevent onward transmission of HIV [2-4], children and adolescents suffer high rates of treatment failure: among those younger than 15 years who receive ART, reported rates of treatment failure in Eastern and Southern Africa range from 10\% (Eswatini) to over 50\% (Eritrea, Mozambique, South Sudan) [5].

Treatment failure can be caused by non-adherence to therapy, viral drug resistance, or a combination of both, requiring differentiated clinical management. Without resistance testing, healthcare providers cannot definitively determine whether treatment failure is caused by drug resistance, necessitating an urgent switch of drug regimen, or non-adherence, in which case underlying causes must be addressed and unnecessary switching must be avoided to preserve the limited future treatment options.

Access to genotypic resistance testing (GRT) to detect viral drug resistance is lacking in most low-income settings [6]. As national HIV programs in sub-Sahara Africa struggle with limited resources, the question if resistance testing is of real clinical benefit or rather a "nice to have" is important as it impacts resource allocation within programs. The World Health Organisation (WHO) recommends resistance testing only upon confirmed treatment failure on second-line ART and/or proteaseinhibitor-based ART, and even then only after a lengthy process of enhanced adherence counselling followed by a confirmatory viral load (VL) test [7].

A recent systematic review on the impact of genotypic and/or phenotypic resistance testing in ART-experienced individuals only found randomised clinical trials published before 2007, all conducted in Europe, the USA, or South America, only two of which included children and/or adolescents [8]. This review reported a potential slight reduction of virologic failure where resistance testing was available, but little or no difference in mortality, CD4 cell count, progression to AIDS, or adverse events. Among three modelling studies on the cost-effectiveness of GRT in southern Africa, published between 2011 and 2014, conclusions differed greatly [9-11].

Three ongoing randomised clinical trials (in addition to the trial presented here) will assess the usefulness of resistance testing in sub-Saharan Africa: the REVAMP study, conducted in South Africa and Uganda, is assessing the feasibility, effectiveness, and cost-effectiveness of GRT upon detection of viremia in adults taking nonnucleoside reverse transcriptase inhibitor- (NNRTI)based first-line ART [12]. A trial in Tanzania, including all age groups, implements GRT upon confirmation of treatment failure after enhanced adherence counselling [13]. Finally, the Opt4Kids trial assesses the impact of a combination of point-of-care VL testing and targeted resistance testing among children on first-line ART in Kenya [14].

We report here the protocol of the trial: Genotype-Informed Versus Empiric Management Of VirEmia (GIVE MOVE) in HIV-Infected Children and Adolescents on Antiretroviral Therapy: An Open-Label Randomised Clinical Trial. GIVE MOVE is among the first randomised clinical trials assessing the clinical impact of providing GRT to children and adolescents with viremia while on first-line ART, with key differences in study design compared to the above-mentioned ongoing trials.

\section{Methods}

Aim

The GIVE MOVE trial assesses whether timely provision of GRT upon detection of viremia improves health outcomes for children and adolescents on first-line ART 
when compared to the current standard of care. In the case of an observed clinical benefit, the costeffectiveness of this intervention will be assessed. Combined, these results will provide evidence on whether the availability of GRT should be prioritised for children and adolescents living with HIV in resource-limited settings.

\section{Design and study setting}

GIVE MOVE is a multi-centre, parallel-group (1,1 allocation), open-label, superiority randomised clinical trial conducted in Lesotho and Tanzania. These two countries are home to 21,000 and 150,000 children and adolescents living with HIV [1], respectively, and have a reported adult HIV prevalence of $22.8 \%$ in Lesotho and $4.8 \%$ in Tanzania [5].

Enrolment will take place at four sites. In Lesotho, these are the Satellite Centres of Excellence of the Baylor College of Medicine Children's Foundation Lesotho ('Baylor Clinics') located in Hlotse, Butha-Buthe, and Mokhotlong. In Tanzania, the study is conducted at the One-Stop Clinic of the Chronic Diseases Clinic Ifakara at Saint Francis Referral Hospital in Ifakara, Kilombero District. In both countries, additional sites have been identified for potential inclusion at a later stage.

GRT takes place at the laboratory of Seboche Mission Hospital in Butha-Buthe district, Lesotho and at the laboratory of the Ifakara Health Institute in Ifakara, Tanzania. All other laboratory diagnostics are conducted at laboratories associated with the respective sites.

\section{Participants}

Potential participants are identified through prescreening of routine medical records.

Inclusion criteria are: being in care in a study site; age $\geq 6$ months and $<19$ years; latest HIV VL result $\geq 400$ copies $/ \mathrm{mL}(\mathrm{c} / \mathrm{mL})$; being on a first-line ART regimen (defined as never having had a regimen change due to virologic failure); having been on an unchanged ART regimen for $\geq 6$ months; phlebotomy for the latest VL test done < 3 months before screening; and written informed consent.

Exclusion criteria are: an indication for treatment switch according to WHO guidelines at screening; initiation of the first session of enhanced adherence counselling > 2 weeks prior to screening; an intention to transfer out of the study site (and not into a different study site) within 3 months after randomisation; already being enrolled in another study if judged as noncompatible by the (Local) Principal Investigator; being pregnant or breastfeeding at screening (no exclusion based on pregnancy or breastfeeding after enrolment); acute illness requiring hospitalisation at screening (no exclusion based on hospitalisation after enrolment); and having received an HIV resistance test in the last 12 months.

\section{Consent procedures, screening, and randomisation}

Consent is provided by the participant if aged $\geq 16$ years (Lesotho) or $\geq 18$ years (Tanzania), and by the caregiver for younger participants. Minors aged $\geq 6$ years additionally receive age-appropriate study information and provide informed assent. Written informed consent and, where applicable, written informed assent is a prerequisite for participation in this study. Details on consenting procedures are listed in the declarations below. Formal screening, including a non-routine pregnancy test for female adolescents aged $\geq 12$ years, takes place only after consent (and assent, if applicable) has been obtained.

Eligible and consenting individuals are enrolled and randomised in a 1:1 ratio to the intervention and control arms. Randomisation is stratified by country (Lesotho or Tanzania), age at enrolment ([ $\geq 6$ months to $<12$ years $]$ or $[\geq 12$ years to $<19$ years]), and type of ART regimen at enrolment (NNRTI-, protease inhibitor- (PI-), or integrase strand transfer inhibitor- (INSTI-)based regimen), using permuted blocks with varying block size. Randomisation is automated using the electronic data capture software MACRO version 4.8.1 (Elsevier) once eligibility and consent have been confirmed and entered into the database, thereby maintaining concealment of allocation.

\section{Control and intervention arm}

The control arm is largely based on the WHO and national guidelines $[7,15,16]$ : Participants in the control arm receive (at least) three sessions of adherence counselling followed by a second VL test. Onward treatment is determined by the outcome of this second VL test: sustained viremia $\geq 400 \mathrm{c} / \mathrm{mL}$ triggers a switch to a second-line ART regimen selected based on empiric criteria according to national guidelines $[15,16]$, whereas viral resuppression to $<400 \mathrm{c} / \mathrm{mL}$ results in continuation of the current regimen.

This cut-off of $400 \mathrm{c} / \mathrm{mL}$ for viral suppression was selected based on the growing body of evidence that the cut-off of $1000 \mathrm{c} / \mathrm{mL}$ currently recommended by the WHO [7] and the national guidelines of the project countries $[15,16]$ may be too high $[17-21]$. While guidelines suggest the confirmatory VL test should be delayed and adherence counselling should continue in the case of 'ongoing poor adherence', the GIVE MOVE protocol allows for this only upon evidence of nonadherence defined as i) a pill count $<90 \%$, or ii) a selfreported period of no drug intake of $\geq 2$ days during the past 4 weeks.

Participants in the intervention arm receive an intervention package consisting of: i) GRT by Sanger sequencing completed by an in-country laboratory (target 
turn-around time: 2 weeks); ii) review of the GRT result by at least three members of a GRT Expert Committee, providing a recommendation for onward treatment (target turn-around time: 1 week); iii) GRT-informed choice of onward therapy; and iv) GRT-informed further adherence counselling.

The main study visits for each arm are shown in Fig. 1. Additionally, a ' 6 months post decision visit' takes place 24 weeks (range: $20-28$ weeks) after the decision on onward therapy, i.e. after the visit in which the follow-up VL result (control arm) or the GRT result (intervention arm) become available. Depending on the timing, this ' 6 months post decision visit' is either combined with another study visit or conducted separately. Any additional visits and laboratory tests taking place according to the standard of care or clinical necessity (including but not limited to: more frequent clinical visits upon pregnancy; check-up visits after modifications to the ART regimen; clinical indication) are recorded. Missing participants will be traced, contacted and encouraged to return back to care. The study procedures at each study visit are shown in the SPIRIT diagram in Fig. 2.

\section{Endpoints}

The composite primary endpoint is the occurrence of any one or more of the events i) death due to any cause during the follow-up period ( 36 weeks), ii) HIV- or ART-related hospital admission of $\geq 24 \mathrm{~h}$ duration (possibly, probably or definitely related to HIV or ART, judged by the endpoint committee blinded to the study arm) during follow-up, iii) new clinical WHO stage IV event (excluding lymph node tuberculosis, stunting, oral or genital herpes simplex infection and oesophageal candidiasis; judged by the endpoint committee blinded to the study arm) during follow-up, and iv) no documentation of a suppressed VL $(<50 \mathrm{c} / \mathrm{mL})$ at 36 weeks followup (window: 32-44 weeks).

The secondary and exploratory endpoints are listed in Table 1.

The first four secondary endpoints are the individual components of the composite primary endpoint.

\section{Sample size}

We hypothesise that $35 \%$ of participants in the control arm will reach the primary endpoint. With $\alpha=0.05$ and $80 \%$ power, a sample size of 276 participants is needed to detect a clinically relevant reduction in the primary endpoint of $15 \%$ in the intervention arm.

\section{Planned analyses}

Analysis and reporting will follow CONSORT guidelines [22] and intention-to-treat (ITT) principles including all participants as randomised. In addition, a per-protocol analysis of the primary endpoint will include all randomised participants who completed the study without a protocol violation. A flowchart will describe the inclusion and follow-up of participants by study arm. Baseline characteristics will be described by study arm with summary statistics such as median and interquartile range or number and percentage; no formal testing between arms will be performed [23]. The primary endpoint as well as categorical secondary and exploratory endpoints will be assessed using a logistic regression model, reporting odds ratios and risk differences with standard errors estimated using the delta method [24]. The exploratory endpoint of time until documented viral suppression will be assessed with Cox proportional hazard models, reporting hazard ratios. All estimates will be reported with 95\% confidence intervals. All models will be adjusted for the stratification factors of country, age, and ART regimen at enrolment. Subgroup analyses are planned by country (Lesotho or Tanzania), sex (female or male), age ([ $\geq 6$ months to $<12$ years] or $[\geq 12$ years to $<19$ years]), and ART regimen at enrolment (NNRTI-, PI-, or INSTI-based regimen).

\section{Interim analysis}

An interim analysis for efficacy and inefficacy is planned once 138 participants (50\% of the target number of participants) have completed the 9-month study visit and/ or reached the primary endpoint. The trial may be concluded early for success if a significant difference between the study arms is achieved for the composite primary endpoint. We will use the conservative Haybittle-Peto stopping level of $p=0.001$ [25]. The trial may be stopped for inefficacy if the odds ratio is greater than 1 and the two-sided 95\% confidence interval does not contain the alternative hypothesis (i.e., odds ratio of $0.57,[26])$. More details are provided in the statistical analysis plan.

The interim analysis will be conducted by an independent statistician. The results will be reviewed by a Data Safety Monitoring Board, who will issue a recommendation to continue or stop the trial to the Steering Committee. For this recommendation, additional information (i.e. new emerging evidence) may also be taken into account. The Steering Committee will vote on and thereby determine the continuation or termination of the trial. In the event of a tie, the Sponsor/Chief Investigator will cast the deciding vote. If the decision is taken to stop the trial, recruitment will be suspended but participants who are already enrolled will continue to be followed for the primary and secondary outcomes.

\section{Data collection and management}

Data is captured online in electronic case report forms in the password-protected MACRO database, which generates an audit trail. On the electronic report forms, 


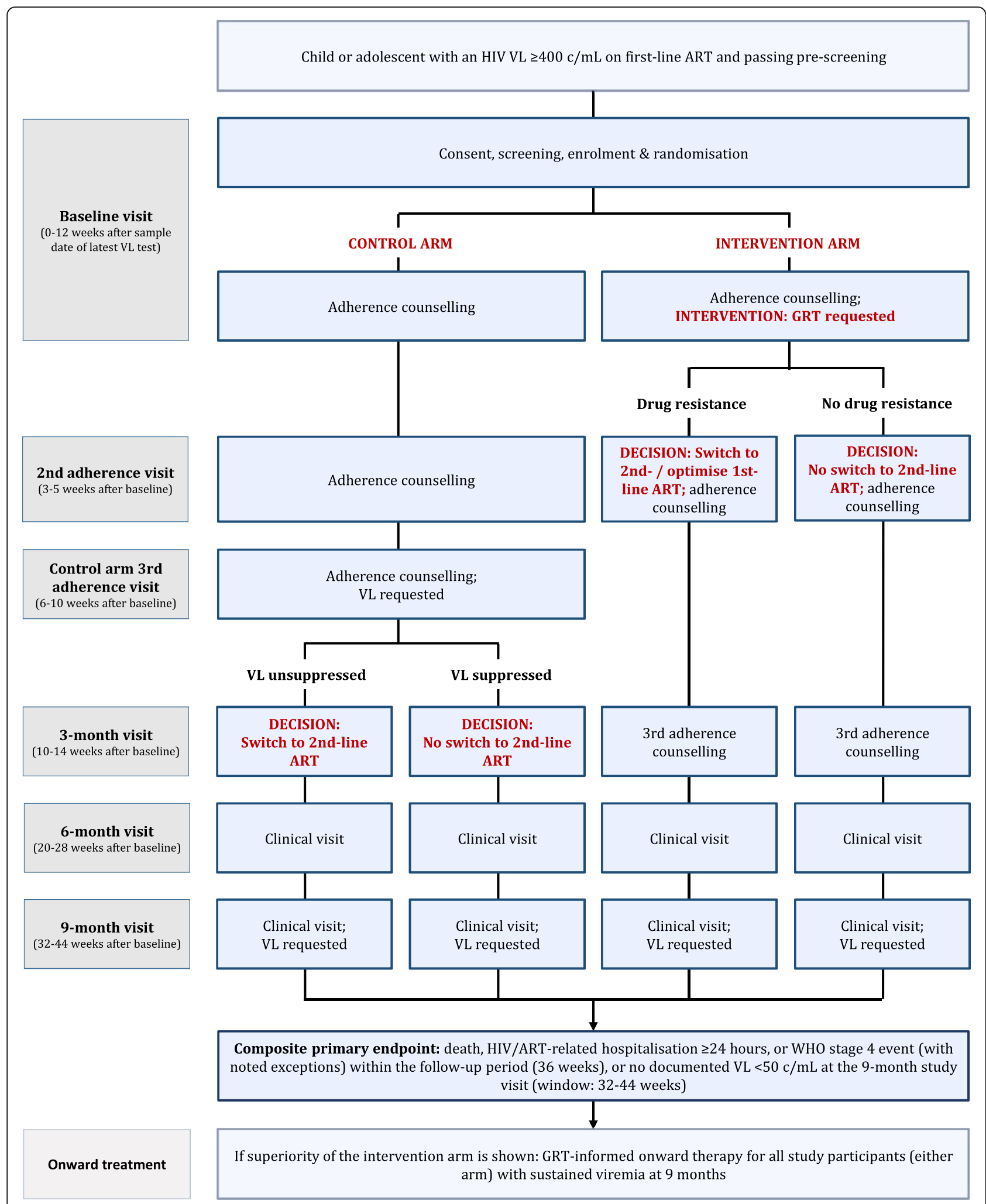

Fig. 1 Flow chart of GIVE MOVE treatment algorithm and study visits. Detailed procedures at each study visit are listed in Fig. 2. GRT: genotypic resistance testing; VL: viral load 


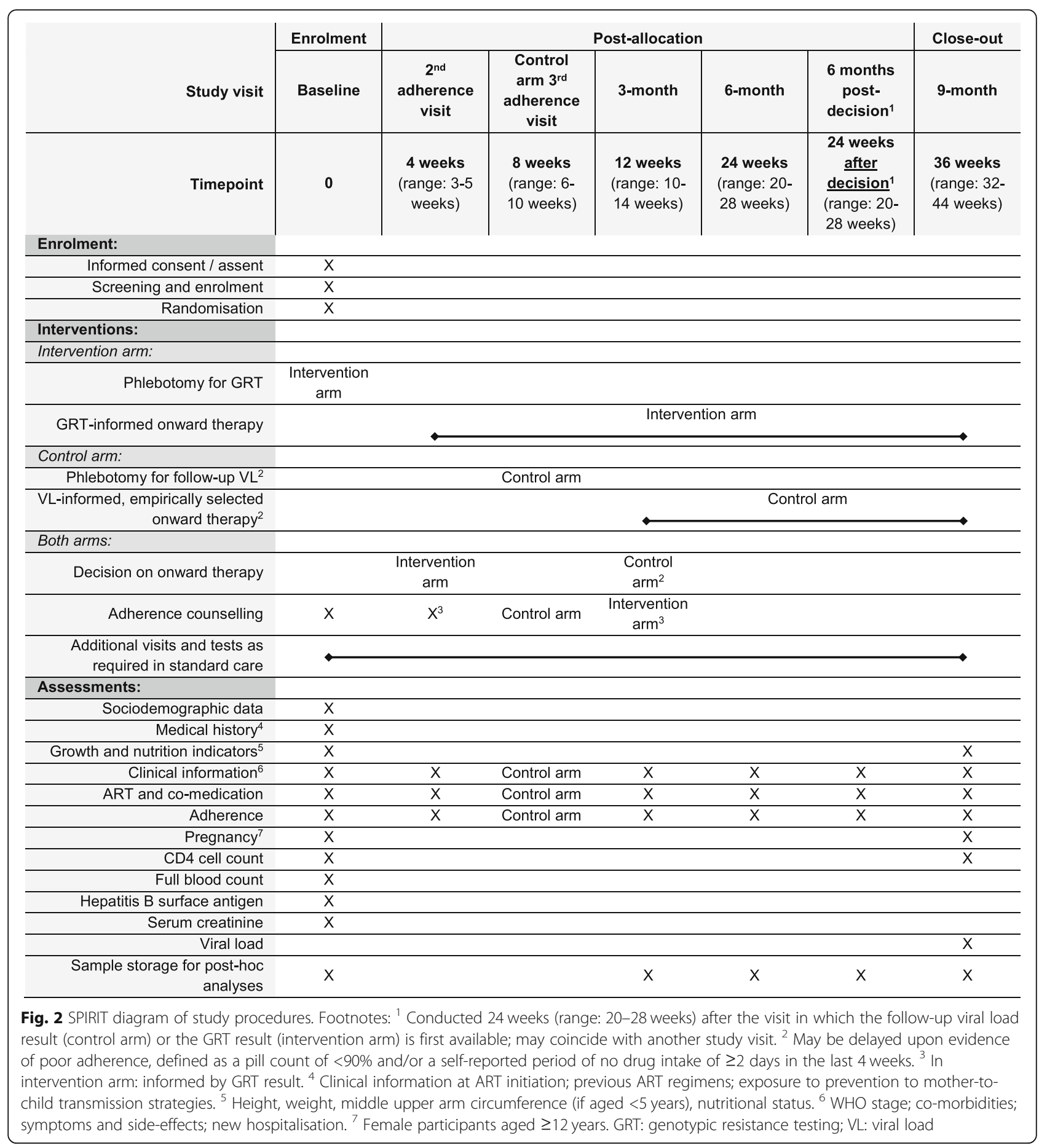

participants are identified by a unique identifier and no participant names are stored in the database. A paperbased participant identification list, the Informed Consent/Assent Forms, and paper-based source documents are kept under lock and key at each study site. All study data and documentation will be archived for at least 10 years after completion of the study.

\section{Plasma collection and storage}

Participants will undergo phlebotomy at enrolment and 2 (control arm only), 3, 6 and 9 months after enrolment (see Spirit diagram in Fig. 2). Study-related phlebotomy will be limited to age-appropriate volumes per blood draw, defined as $\leq 5 \mathrm{~mL}$ for participants $<5$ years; $\leq 10 \mathrm{~mL}$ for participants $\geq 5$ and $<10$ years; $\leq 15 \mathrm{~mL}$ for 
Table 1 Secondary and exploratory endpoints

Endpoint
Secondary endpoints
Death due to any cause
HIV- or ART-related hospitalisation of $\geq 24 \mathrm{~h}$
duration

New clinical WHO stage 4 event(s)

Without documentation of a suppressed $\mathrm{VL}$

Loss to follow-up

Observed virologic failure

Composite endpoint at 6 months after the decision on onward treatment

\section{Exploratory endpoints}

Time to documented viral suppression

Drug regimen switches in the absence of major resistance-associated mutations and/or nonswitches in the presence of major resistanceassociated mutations

Proportion with new resistance-associated mutations emerged within the study period

\section{Definition}

Proportion of participants confirmed dead during the follow-period among all participants enrolled

Proportion of participants with HIV- or ARTrelated hospital admission(s) of $\geq 24 \mathrm{~h}$ duration (possibly, probably or definitely related to HIV or ART, judged by the endpoint committee blinded to the study arm) during the follow-up period among all participants enrolled

Proportion of participants with new clinical WHO stage 4 event(s) (excluding lymph node tuberculosis, stunting, oral or genital herpes simplex infection and oesophageal candidiasis, judged by the endpoint committee blinded to the study arm) among all participants enrolled

Proportion of participants without documentation of a $\mathrm{VL}<50 \mathrm{c} / \mathrm{mL}$ at 9 months among all participants enrolled

Proportion of participants with no documented clinic visit at 9 months among all participants enrolled

Proportion of participants with a $\mathrm{VL} \geq 50 \mathrm{c} / \mathrm{mL}$ among all participants with a VL result at 9 months

Proportion of participants among all participants enrolled experiencing any one or more of the events i) death due to any cause within 24 weeks of the decision on onward treatment, ii) HIV- or ART-related hospital admission of $\geq 24 \mathrm{~h}$ duration (possibly, probably or definitely related to HIV or ART, judged by the endpoint committee blinded to the study arm) within 24 weeks of the decision on onward treatment, iii) new clinical WHO stage IV event (excluding lymph node tuberculosis, stunting, oral or genital herpes simplex infection and oesophageal candidiasis; judged by the endpoint committee blinded to the study arm) within 24 weeks of the decision on onward treatment, and iv) no documentation of a suppressed $\mathrm{VL}(<50 \mathrm{c} / \mathrm{mL})$ at 6 months (20-28 weeks) after the choice of onward treatment. The time point of the decision on onward treatment is defined as the first visit in which the follow-up VL result (control arm) or the GRT result (intervention arm) is available.

Time to achieving a $\mathrm{VL}<50 \mathrm{c} / \mathrm{mL}$; considering $\mathrm{VL}$ testing done with samples from the 3-, 6- and 9month study visits in both arms

Proportion of participants with ART regimen switches in the absence of major resistanceassociated mutations and/or non-switches in the presence of major resistance-associated mutations among all participants enrolled (as identified by Sanger sequencing, according to the Stanford HIV drug resistance database).

Proportion of participants with new resistanceassociated mutations emerged within the study period among all participants enrolled

\section{Timeframe}

Within 36 weeks after enrolment

Within 36 weeks after enrolment

Within 36 weeks after enrolment

(1)

32—44 weeks after enrolment

32-44 weeks after enrolment

32-44 weeks after enrolment

i-iii): within 24 weeks after the decision on onward therapy;

iv): 20 - 28 weeks after the decision on onward therapy
Assessed at 3- (10-14 weeks after enrolment), 6- (20-28 weeks after enrolment), and 9-month study visit (32-44 weeks after enrolment)

Assessed at enrolment and at 9-month study visit (32-44 weeks after enrolment)

Change from enrolment to 9-month study visit (32-44 weeks after enrolment) 
participants $\geq 10$ and $<15$ years; and $\leq 25 \mathrm{~mL}$ for participants $\geq 15$ years [27]. The sites receive training and written guidance on the safe blood volumes in paediatric patients and the prioritisation of laboratory procedures in the event that insufficient blood is available to perform all tests as per protocol.

Biological material is identified by the participant's study ID and processed or stored at $-80^{\circ} \mathrm{C}$ at the laboratory site in charge. Consent is collected for further use of samples in future studies, subject to approval from the relevant ethics committee(s).

\section{Monitoring}

In Lesotho, GIVE MOVE is monitored by the Monitoring Group of the Clinical Operations Unit at the Swiss Tropical and Public Health Institute, as well as monitors from SolidarMed Lesotho who are supervised by this group. In Tanzania, the trial is monitored by the Ifakara Health Institute. The first two participants per site and approximately $10 \%$ of the total number of participants will undergo $100 \%$ source data verification. The remaining participants will undergo source data verification of all key data as defined in the monitoring plan. For each study site a site initiation visit, regular routine monitoring visits and a close-out visit are planned.

In addition, the Baylor Clinics are audited on a halfyearly basis by the Baylor College of Medicine Children's Foundation Lesotho.

\section{Ethical considerations}

This study has been approved by the relevant ethics committees and, if applicable, other authorities in the project countries. In addition, a Swiss ethics committee provided a statement confirming the trial meet ethical requirements. Details are listed in the declarations below.

All participants/caregivers are informed that participation is voluntary and that they may withdraw from the study at any time. Participants do not receive any form of remuneration, though transport costs to the study site are compensated for participants and up to one caregiver.

Pregnancy (assessed by a pregnancy test in female adolescents aged $\geq 12$ years during screening) or breastfeeding at enrolment are exclusion criteria; however, pregnancy after enrolment does not lead to exclusion. Participants who become pregnant during the study period will receive additional services including more frequent visits and additional laboratory testing in accordance with the national guidelines $[15,16]$. If births occur during the study period, the HIV status and health of the new-born will be recorded.

The following serious adverse events will be captured and reported to the ethics committees: any untoward medical occurrence that i) results in death or is lifethreatening; ii) requires in-patient hospitalisation or prolongation of existing hospitalisation; iii) results in persistent or significant disability or incapacity; or iv) causes a congenital anomaly or birth defect.

In the case that the intervention proves beneficial, all participants will receive GRT if they still have an elevated VL at study closure.

\section{Nested study on cost-effectiveness}

A nested study will assess the cost and cost-effectiveness of GRT. For this purpose, the number of clinical visits and care received at each visit (e.g. counselling, clinical exam with a doctor/nurse), the number and duration of hospitalisations, concomitant medication, and all requested laboratory tests are recorded for each participant.

\section{Trial registration}

This trial has been registered with ClinicalTrials.gov (NCT04233242; registered 18.01.2020; https://clinicaltrials.gov/ct2/show/NCT04233242). Further information is available on the trial website: www.givemove.org.

\section{Discussion}

Evidence to guide the management of treatment failure in children and adolescents in low-income settings is desperately lacking, endangering the UNAIDS vision of an AIDS-free generation by 2030 [28].

Early, successful ART is key to child development as it reduces mortality and morbidity, improves neurocognitive and growth outcomes $[29,30]$, and preserves future therapeutic options. However, intention-to-treat analyses report that $20-30 \%$ of children and adolescents have an unsuppressed VL 1 year after starting first-line ART [31, 32]. Similarly, a recent systematic review showed that after undergoing enhanced adherence counselling upon detection of viremia, subsequent resuppression was achieved by a little over half (50.4\%) of adults, but only 31.2 and $40.4 \%$ of children and adolescents, respectively [33]. Even among children whose ART regimen is switched to second-line, resuppression rates remain low [34].

The GIVE MOVE trial assesses the feasibility, clinical impact, and cost-effectiveness of GRT to guide the clinical management of viremia despite first-line ART in children and adolescents. We hypothesise that GRT will substantially improve treatment outcomes by allowing for differentiated care customised according to the individual child/adolescent's health situation and needs, i.e. targeted adherence support for those without drug resistance and a rapid switch to an optimised ART regimen (with potential additional adherence support) in those with drug-resistant HIV, as well as by reducing the time to appropriate clinical action. 
This trial has several limitations. Given the nature of the intervention, blinding of participants or healthcare professionals is not possible. Furthermore, the trial cannot make full use of the potential of GRT to reduce time to clinical decision-making: due to the ethical necessity of consenting participants before conducting any nonroutine procedures, enrolment and phlebotomy for GRT take place ideally at the first clinic visit after a routine VL test (generally after 1 month). In clinical practice, however, it would be possible to use blood remaining after VL testing to immediately conduct GRT if viremia is detected, and provide both the VL and the GRT result at the subsequent clinic visit. Thus, the GIVE MOVE trial will likely underestimate the potential benefit of reducing time to clinical decision-making.

However, this trial also has several strengths. The multi-site approach, as well as the fact that this pragmatic trial relies heavily on existing infrastructure at the study sites and the logistical capacities of the in-country partners, will increase external validity. The inclusion of participants on newer INSTI-based ART regimens (notably dolutegravir-based regimens) ensures that results will remain relevant for years to come as dolutegravirbased ART becomes increasingly available [35], and the focus on children and adolescents ensures that the needs of particularly vulnerable age groups are addressed.

In conclusion, the GIVE MOVE trial will assess if the availability of GRT for children and adolescents with unsuppressed VLs while taking ART improves clinical outcomes and if it is cost-effective. While funding for national HIV programs in Africa is stagnating or even decreasing, it is more important than ever that resource allocation gives highest priority to evidence-based interventions. Data from GIVE MOVE will provide evidence to program managers and policymakers for the decision on whether access to GRT is an intervention to which further resources should be allocated.

\section{Trial status}

The trial was launched at the first site (Baylor Clinic Hlotse) on 20.02.2020, and the first participant was enrolled on 03.03.2020. As per 06.10.2020, all sites are following study protocol v1.3 (dated 27.02.2020), 33 participants have been enrolled, and all four sites have enrolled at least one participant. Enrolment is expected to continue until mid- to late 2021, with a subsequent follow-up period of up to 11 months.

\footnotetext{
Abbreviations

ART: Antiretroviral therapy; c/mL: Copies per millilitre; GIVE MOVE: GenotypeInformed Versus Empiric Management Of VirEmia; GRT: Genotypic resistance testing; HIV: Human immunodeficiency virus; INSTI: Integrase strand transfer inhibitor; ITT: Intention-to-treat; NNRTI: Non-nucleoside reverse transcriptase inhibitor; PI: Protease inhibitor; VL: Viral load; WHO: World Health Organisation
}

\section{Acknowledgements}

We acknowledge Soheila Aghlmandi, who will perform the interim analysis; Alexandra Calmy, Nathan Ford, Abubakary Mziray, Andreas Schötzau, and Aneth Vedastus Kalinjuma, who constitute the DSMB; Laetitia Kampiire, Amy Slogrove, Ute Feucht, Kristina Keitel, Nicole Ritz, and Noemi Gessler, who constitute the Endpoint Committee; as well as Huldrych Günthard, NDL, TK, and MW who constitute the GRT Expert Committee. We would furthermore like to acknowledge the trial monitors at the Clinical Operations Unit of the Swiss Tropical and Public Health Institute (Sonja Bernhard, Elisabeth Reus, Jarmila Hanekova), SolidarMed (Lipontso Motaboli), and the Ifakara Health Institute (Rose Minja, Beatrice James, Alwisa Urassa). We thank Bruce Larson for his advice on data collection for the cost-effectiveness analysis, and Blaise Lukau for clinical support and coordination. We further acknowledge all team members at the study sites in Hlotse (Mamonyake Mokete, Moliehi Pii, Palesa Mothibeli, and former team member Kaleu Claude Sambayi), Butha-Buthe (LK, 'Matšiu Ralitapole, and Mamello Lebitsa), Mokhotlong (CWDY, Mamotebang Malapane, and Makeletso Nkune) and Ifakara (EL, GJM, and Jenifa Tarimo). Our thanks also go to the laboratory technologists in the GRT laboratories (notably MC and Relebohile Belempi in Lesotho, and NK and Dorcas Mnzava in Tanzania), as well as all further diagnostic personnel in the on-site laboratories. We thank SolidarMed Lesotho for extensive support in terms logistics and human resources. Finally, we gratefully acknowledge the children and adolescents participating in this trial as well as their caregivers.

\section{Authors' contributions}

$\mathrm{NDL}$ is the sponsor/chief investigator, first conceptualised this trial, and acquired funding. JAB is the principal investigator, wrote the first version of the study protocol and this manuscript, and acquired funding. NDL and JAB designed this trial, with significant input from TRG, TK, MW, AA, NB, JM, EL, and GJM. TRG is the trial statistician and responsible for the statistical analysis plan. $M B$ is the principal data manager. IR is the study manager overseeing recruitment and data quality. BM provides expertise and operational support on quality assurance and documentation. JM, BPK, MMH, LT, MW, and DHP provide expertise and coordinating support. MC and NK provide diagnostic expertise and support, and are responsible for GRT in Lesotho and Tanzania, respectively. TK provides further diagnostic expertise. EL, LK, and CWDY are local principal investigators. NDL, JAB, JM, BPK, MMH, IR, MW, EL, TK and TRG form the GIVE MOVE Steering Committee. The authors read and approved the final manuscript

\section{Funding}

This project is primarily funded by the Fondation Botnar (grant number REG19-008; awarded to JAB and NDL). Significant additional funding was provided by the Swiss National Science Foundation (grant number PCEF P3_181355; awarded to NDL). This study is embedded in the SolidarMed country programme in Lesotho and benefits from logistics and human resources provided by SolidarMed in Lesotho and the Ifakara Health Institute in Tanzania. The funders had no role in the design of the study, writing of the study protocol or the decision to submit the study protocol manuscript for publication. They have and will have no role in data collection, data analysis, interpretation of the data, writing of manuscripts, or the decision to submit future manuscripts for publication.

\section{Availability of data and materials}

Results of this research will be disseminated at the district and/or national level to stakeholders within the project countries, as well as at the international level through peer-reviewed publications and academic conferences.

Upon publication of the trial results, a subset of the key pseudo-anonymised individual participant data collected during the study, along with a data dictionary, will be made available through the data repository Zenodo. Case report forms and other key study documents will also be made available upon publication of results. The full dataset will be made available upon request to the Department of Medicine at the Swiss Tropical and Public Health Institute and after signing a data confidentiality agreement.

\section{Ethics approval and consent to participate}

This trial has been approved by the National Health Research Ethics Committee in Lesotho (ID229-2019), the Institutional Review Board of the Ifakara Health Institute (12-2020), the National Institute for Medical Research in Tanzania (NIMR/HQ/R.8a/Nol IX/3442), and the Tanzania Medicines and 
Medical Devices Authority (TMDA0020/CTR/0003/03). In addition, the Ethikkommission Nordwest- und Zentralschweiz in Switzerland provided a statement confirming the trial meets all requirements for a Swiss research project (Req-2019-01275).

Written informed consent and, where applicable, written informed assent is a prerequisite for participation in this study. The consenting process is conducted by an authorised member of the study team based at the respective site. Before deciding whether or not to consent, participants/ caregivers are given detailed information about the study, and are informed that they will continue to receive the standard of care if they choose not to participate and that they may withdraw their consent at any time. Participants aged $\geq 16$ years (Lesotho) or $\geq 18$ years (Tanzania) fill in an Informed Consent Form. For children and younger adolescents below these age thresholds, a caregiver fills in a separate Informed Consent Form for caregivers. Within the approved study protocol, a caregiver is defined as a parent, a legal guardian, or an adult aged $\geq 18$ years and living in the same household as the participant. In addition, participants aged $\geq 12$ and $<16$ years (Lesotho) or $\geq 12$ and $<18$ years (Tanzania) fill in an Informed Assent Form for Adolescents, and those aged $\geq 6$ and $<12$ years fill in a simplified Informed Assent Form for Children. All forms are available in English as well as Sesotho (Lesotho) or Swahili (Tanzania). In the case of illiteracy, consent/ assent is given by thumb print, and a witness signature is required.

\section{Consent for publication}

Not applicable.

\section{Competing interests}

TK reports advisory board membership fees from ViiV and Gilead for work outside of this study. All other authors declare that they have no competing interests.

\section{Author details}

${ }^{1}$ Swiss Tropical and Public Health Institute, Basel, Switzerland. ${ }^{2}$ Molecular Virology, Department of Biomedicine, University of Basel, Basel, Switzerland. ${ }^{3}$ University of Basel, Basel, Switzerland. ${ }^{4}$ SolidarMed, Partnerships for Health, Maseru, Lesotho. Ifakara Health Institute, Ifakara, Tanzania. ${ }^{6}$ Seboche Mission Hospital, Seboche, Lesotho. 'Baylor College of Medicine Children's Foundation Lesotho, Maseru, Lesotho. ${ }^{8}$ Baylor College of Medicine, Houston, TX, USA. ${ }^{9}$ Department of Infectious Diseases and Hospital Epidemiology, University Hospital Basel, Basel, Switzerland.

\section{Received: 20 August 2020 Accepted: 8 October 2020}

\section{Published online: 19 October 2020}

\section{References}

1. UNICEF. Key HIV epidemiology indicators for children and adolescents aged 0-19, 2000-2018; 2019. https://data.unicef.org/wp-content/uploads/2019/ 07/HIV_Epidemiology_Children_Adolescents-2019.xIsx. Accessed 26 Mar 2020.

2. Bavinton BR, Pinto AN, Phanuphak N, Grinsztejn B, Prestage GP, ZablotskaManos IB, et al. Viral suppression and HIV transmission in serodiscordant male couples: an international, prospective, observational, cohort study. Lancet HIV. 2018;5:e438-47.

3. Rodger AJ, Cambiano V, Bruun T, Vernazza P, Collins S, van Lunzen J, et al. Sexual activity without condoms and risk of HIV transmission in serodifferent couples when the HIV-positive partner is using suppressive antiretroviral therapy. JAMA. 2016;16:171-81.

4. Cohen MS, Chen YQ, McCauley M, Gamble T, Hosseinipour MC, Kumarasamy N, et al. Prevention of HIV-1 infection with early antiretroviral therapy. N Engl J Med. 2011;365:493-505.

5. UNAIDS. UNAIDS data 2020. Geneva: UNAIDS; 2020

6. Inzaule SC, Ondoa P, Peter T, Mugyenyi PN, Stevens WS, de Wit TFR, et al. Affordable HIV drug-resistance testing for monitoring of antiretroviral therapy in sub-Saharan Africa. Lancet Infect Dis. 2016;16:e267-75.

7. World Health Organisation. Consolidated guidelines on the use of antiretroviral drugs for treating and preventing HIV infection. Recommendations for a public health approach. 2nd ed. Geneva: WHO; 2016. http://apps.who.int/iris/bitstream/handle/10665/208825/97892415496 84_eng.pdf?sequence $=1$.
8. Aves T, Tambe J, Siemieniuk RA, Mbuagbaw L. Antiretroviral resistance testing in HIV-positive people. Cochrane Database Syst Rev. 2018;11: CD006495.

9. Levison JH, Wood R, Scott CA, Ciaranello AL, Martinson NA, Rusu C, et al. The clinical and economic impact of genotype testing at first-line antiretroviral therapy failure for HIV-infected patients in South Africa. Clin Infect Dis Off Publ Infect Dis Soc Am. 2013;56:587-97.

10. Rosen S, Long L, Sanne I, Stevens WS, Fox MP. The net cost of incorporating resistance testing into HIV/AIDS treatment in South Africa: a Markov model with primary data. J Int AIDS Soc. 2011;14:24.

11. Phillips A, Cambiano V, Nakagawa F, Mabugu T, Magubu T, Miners A, et al. Cost-effectiveness of HIV drug resistance testing to inform switching to second line antiretroviral therapy in low income settings. PLoS One. 2014;9: e109148.

12. Siedner MJ, Bwana MB, Moosa M-YS, Paul M, Pillay S, McCluskey S, et al. The REVAMP trial to evaluate HIV resistance testing in subSaharan Africa: a case study in clinical trial design in resource limited settings to optimize effectiveness and cost effectiveness estimates. HIV Clin Trials. 2017;18:149-55.

13. ClinicalTrials.gov. Impact of HIV drug resistance testing, and subsequent change to an individualized therapy in Tanzania; 2018. https://clinicaltrials. gov/ct2/show/NCT03557021. Accessed 18 Jan 2019.

14. ClinicalTrials.gov. Optimizing viral load suppression in Kenyan children on antiretroviral therapy; 2019. https://clinicaltrials.gov/ct2/show/NCT03820323. Accessed 16 Aug 2020.

15. Ministry of Health, Government of Lesotho. National Guidelines on the use of antiretroviral therapy for HIV prevention and treatment (5th edition); 2016.

16. Ministry of Health, Community Development, Gender, Elderly, and Children. Tanzania national guidelines for the management of HIV and AIDS. 6th ed. Dar es Salaam: Ministry of Health, Community Development, Gender, Elderly, and Children; 2017.

17. Kantor R, DeLong A, Schreier L, Reitsma M, Kemboi E, Orido M, et al. HIV-1 second-line failure and drug resistance at high-level and low-level viremia in Western Kenya. AIDS Lond Engl. 2018;32:2485-96.

18. Labhardt ND, Bader J, Lejone TI, Ringera I, Hobbins MA, Fritz C, et al. Should viral load thresholds be lowered?: revisiting the WHO definition for virologic failure in patients on antiretroviral therapy in resource-limited settings. Med Baltim. 2016;95:e3985.

19. Gonzalez-Serna A, Min JE, Woods C, Chan D, Lima VD, Montaner JSG, et al. Performance of HIV-1 drug resistance testing at low-level viremia and its ability to predict future virologic outcomes and viral evolution in treatment-naive individuals. Clin Infect Dis Off Publ Infect Dis Soc Am. 2014;58:1165-73.

20. Swenson LC, Min JE, Woods CK, Cai E, Li JZ, Montaner JSG, et al. HIV drug resistance detected during low-level viraemia is associated with subsequent virologic failure. AIDS Lond Engl. 2014;28:1125-34.

21. Amstutz A, Nsakala BL, Vanobberghen F, Muhairwe J, Glass TR, Namane T, et al. Switch to second-line versus continued first-line antiretroviral therapy for patients with low-level HIV-1 viremia: an open-label randomized controlled trial in Lesotho. PLoS Med. 2020;17:e1003325 Accepted; in press.

22. Schulz KF, Altman DG, Moher D, CONSORT Group. CONSORT 2010 statement: updated guidelines for reporting parallel group randomised trials. Trials. 2010;11:32.

23. Pocock SJ, Assmann SE, Enos LE, Kasten LE. Subgroup analysis, covariate adjustment and baseline comparisons in clinical trial reporting: current practice and problems. Stat Med. 2002;21:2917-30.

24. Norton EC, Miller MM, Kleinman LC. Computing adjusted risk ratios and risk differences in Stata. Stata J. 2013;13:492-509.

25. Schulz KF, Grimes DA. Multiplicity in randomised trials II: subgroup and interim analyses. Lancet Lond Engl. 2005;365:1657-61.

26. Freidlin B, Korn EL, Gray R. A general inefficacy interim monitoring rule for randomized clinical trials. Clin Trials Lond Engl. 2010;7:197-208.

27. Howie SRC. Blood sample volumes in child health research: review of safe limits. Bull World Health Organ. 2011;89:46-53.

28. UNAIDS. UNAIDS strategy 2016-2021: on the fast track to end AIDS; 2015. http://www.unaids.org/en/resources/documents/2015/UNAIDS_PCB37_15-18. Accessed 22 Jan 2018.

29. Crowell CS, Huo Y, Tassiopoulos K, Malee KM, Yogev R, Hazra R, et al. Early viral suppression improves neurocognitive outcomes in HIV-infected children. AIDS Lond Engl. 2015;29:295-304. 
30. Shiau S, Arpadi S, Strehlau R, Martens L, Patel F, Coovadia A, et al. Initiation of antiretroviral therapy before 6 months of age is associated with faster growth recovery in south African children perinatally infected with human immunodeficiency virus. J Pediatr. 2013:162:1138-45 1145.e1-2.

31. Jiamsakul A, Kariminia A, Althoff KN, Cesar C, Cortes CP, Davies M-A, et al. HIV viral load suppression in adults and children receiving antiretroviral therapy-results from the leDEA collaboration. J Acquir Immune Defic Syndr 1999. 2017:76:319-29.

32. Boerma RS, Boender TS, Bussink AP, Calis JCJ, Bertagnolio S, Rinke de Wit TF, et al. Suboptimal viral suppression rates among HIV-infected children in low- and middle-income countries: a meta-analysis. Clin Infect Dis Off Publ Infect Dis Soc Am. 2016;63:1645-54.

33. Ford N, Orrell C, Shubber Z, Apollo T, Vojnov L. HIV viral resuppression following an elevated viral load: a systematic review and meta-analysis. J Int AIDS Soc. 2019;22:e25415.

34. Lazarus E, Nicol S, Frigati L, Penazzato M, Cotton MF, Centeno-Tablante E, et al. Second- and third-line antiretroviral therapy for children and adolescents: a scoping review. Pediatr Infect Dis J. 2017;36:492-9.

35. World Health Organisation. Update of recommendations on first- and second-line antiretroviral regimens. Geneva: WHO; 2019.

\section{Publisher's Note}

Springer Nature remains neutral with regard to jurisdictional claims in published maps and institutional affiliations.

Ready to submit your research? Choose BMC and benefit from:

- fast, convenient online submission

- thorough peer review by experienced researchers in your field

- rapid publication on acceptance

- support for research data, including large and complex data types

- gold Open Access which fosters wider collaboration and increased citations

- maximum visibility for your research: over $100 \mathrm{M}$ website views per year

At $\mathrm{BMC}$, research is always in progress.

Learn more biomedcentral.com/submissions 